\title{
Biodegradable Poly(D,L-lactide)/Lipid Blend Microparticles Prepared by Oil-in-Water Emulsion Method for Controlled Release Drug Delivery
}

\author{
YAOWALAK SRISUWAN and YODTHONG BAIMARK*
}

Biodegradable Polymers Research Unit, Department of Chemistry and Center of Excellence for Innovation in Chemistry, Faculty of Science, Mahasarakham University, Mahasarakham 44150, Thailand.

${ }^{*}$ Corresponding author E-mail: yodthong.b@msu.ac.th

http://dx.doi.org/10.13005/ojc/300108

(Received: January 15, 2014; Accepted: March 03, 2014)

\begin{abstract}
The effects of blend ratio and drug loading content of poly(D,L-lactide) (PDLL)/stearic acid blends on microparticle characteristics and drug release behaviors were evaluated. The blend microparticles were prepared by an oil-in-water emulsion solvent evaporation method for drug delivery of a poorly water-soluble model drug, indomethacin. The microparticles were characterized using a combination of scanning electron microscopy (SEM), light scattering particle size analysis, differential scanning calorimetry (DSC) and UV-vis spectrophotometry. The blend microparticles with a PDLL/stearic acid blend ratio in the range 100/0-95/5 (w/w) exhibited a spherical shape with a smooth surface. Blend microparticles with a similar size $(167-177 \mu \mathrm{m})$ and drug loading efficiency $(60-67 \%)$ were obtained. The drug loading content did not affect the characteristics of the blend microparticles. An in vitro drug release test demonstrated that the level of drug release decreased as the stearic acid blend ratio increased and the drug loading content decreased. The overall results indicated that it was possible to use PDLL/stearic acid blend microparticles as a controlled release drug delivery system.
\end{abstract}

Key words: biodegradable polymer, poly(D,L-lactide),

Stearic acid, polymer blends, microparticles, drug delivery.

\section{INTRODUCTION}

Controlled release, prolonged release and sustained release are terms used to refer to drug delivery systems that are produced to deliver precise drug amounts at a pre-programmed rate to achieve the drug level necessary for treatment after administration of the system ${ }^{1,2}$. Controlled release drug delivery also attempts to maintain drug levels within the therapeutic level to reduce potentially hazardous peaks in drug concentration ${ }^{3}$. Biodegradable polymers have been widely used as a matrix for controlled release drug delivery systems in particles, film, fibre and gel forms. The removal of these biodegradable polymeric devices at the completion of therapy is not required. The drug 
release mechanisms of a biodegradable polymeric matrix consist of drug diffusion out and matrix erosion stages.

Amorphous poly(D,L-lactide) (PDLL) is a synthetic biodegradable polymer. This induces uniform drug distribution in the PDLL matrix. PDLL microparticles have been widely investigated as controlled-release drug delivery systems ${ }^{4-7}$. The oilin-water emulsion solvent evaporation technique has been usually used to prepare the PDLL microparticles containing poorly water-soluble model drugs ${ }^{4,7}$. Drug release from polyester particles depended upon the polyester type, polyester molecular weight, drug loading content, particle size and polymer blending ${ }^{8-}$ ${ }^{10}$. Lipids are inexpensive and low toxicity substances that have been widely studied as a matrix for drug carriers $^{11-14}$. Solid lipid microparticles attain high encapsulation efficiency for poorly water-soluble drugs due to their hydrophobic nature. Stearic acid microparticles combine the advantages of liposome and polymer microparticles, while avoiding some of their disadvantages such as toxicity, biodegradability problems and raw material costs ${ }^{15}$. However, PDLL/ stearic acid blend microparticles for use as controlled release drug delivery applications have not been reported.

In this paper, PDLL/stearic acid blend miroparticles containing a poorly water-soluble model drug were prepared by the oil-in-water emulsion solvent evaporation method. The influences of the PDLL/stearic acid blend ratio and drug loading content on morphology, particle size and thermal transition properties of the blend microparticles was determined. In vitro drug release behaviour was also investigated.

\section{EXPERIMENTAL}

\section{Materials}

Poly(D,L-lactide) (PDLL) was synthesized by ring-opening polymerization of a D,L-lactide monomer in bulk under a nitrogen atmosphere at $140^{\circ} \mathrm{C}$ for $24 \mathrm{~h}$ using 1 -dodecanol (98\%, Fluka) and stannous octoate (95\% Sigma) as the initiator and catalyst, respectively. The number-average molecular weight and molecular weight distribution of the resulting PDLL obtained from the GPC method were $17,050 \mathrm{~g} / \mathrm{mol}$ and 1.4, respectively. The 1-dodecanol was distilled under reduced pressure before use. Stannous octoate, stearic acid (95\%, Sigma-Aldrich, USA), indomethacin model drug (99\%, Sigma) and Tween80 (Labchem) were used without further purification. Dichloromethane (Carlo Erba) was used in analytical grade.

\section{Preparation of blend microparticles}

The PDLL/stearic acid blend microparticles containing the model drug were prepared by the oil-in-water emulsion solvent evaporation method. Briefly, $0.1 \mathrm{~g}$ of PDLL/stearic acid mixture was completely dissolved in $2.5 \mathrm{~mL}$ of dichloromethane before pouring into $400 \mathrm{~mL}$ of 2 wt\% Tween80 aqueous solution under magnetic stirring to form an oil-in-water emulsion. The dichloromethane was evaporated in a fume hood for $6 \mathrm{~h}$. The blend microparticles were collected by centrifugation and washed with distilled water three times. The obtained blend microparticles were then freeze-dried overnight and stored in a desiccator before characterization and in vitro drug release testing.

\section{Characterization of blend microparticles}

The morphology of the microparticles was determined by scanning electron microscopy (SEM) using a JEOL JSM-6460LV SEM. The microparticles were coated with gold to enhance conductivity before scanning. The average particle size of the microparticles was measured by light scattering (LS) analysis using a Coulter LS230 particle size analyzer at $25^{\circ} \mathrm{C}$ in a water medium. Thermal transition properties of the microparticles were carried out by means of differential scanning calorimetry (DSC) using a Perkin-Elmer DSC Pyris Diamond. For DSC analysis, $5-10 \mathrm{mg}$ of sample was heated at $10^{\circ} \mathrm{C} /$ min under a helium flow.

The drug loading content of the indomethacin model drug entrapped in the blend microparticles was determined by dissolving $20 \mathrm{mg}$ of blend microparticles in $1 \mathrm{~mL}$ of dichloromethane. The indomethacin concentration in clear solution was determined from the absorbance at $\lambda_{\max }=319 \mathrm{~nm}$ by a UV-Vis spectrophotometry using a Perkin-Elmer Lambda 25 and compared to a standard curve of indomethacin. Theoretical drug loading content $\left(D C_{\text {theoretical }}\right)$, actual drug loading content (DLC $\left.C_{\text {actual }}\right)$ and drug loading efficiency (DLE) were calculated from equations (1)-(3), respectively. The $D C_{\text {actual }}$ is 
an average value from three determinations.

$$
\begin{aligned}
& \operatorname{DLC}_{\text {theoretical }}(\%)=\frac{\text { Weight } 6 \text { feed drug }}{\text { Weight } 6 \text { feed drug, PDLL and stearic acid }} \times 100 \\
& D L C_{\text {actual }}(\%)=\frac{\text { Weight } 6 \text { drug } \text { in blend microparticles }}{\text { Weight } 6 \text { blend microparticles }} \times 100
\end{aligned}
$$

$$
\operatorname{DLE}(\%)=\frac{\mathrm{DLC} \text { actual }}{\mathrm{DLC} \text { theoretical }} \times 100
$$

\section{In vitro drug release test}

An in vitro drug release test was performed in a phosphate buffer solution (PBS, 0.1 M, pH 7.4) at $37^{\circ} \mathrm{C}$ under shaking. The blend microparticles $(\sim 10$ $\mathrm{mg}$ ) were placed in a dialysis tube before immersing in $200 \mathrm{~mL}$ of buffer. At predetermined time intervals, $10 \mathrm{~mL}$ of release medium was withdrawn. Then $10 \mathrm{~mL}$ of fresh buffer was added to the original to maintain the total volume. The drug release was determined by UV-vis spectrophotometry at $\lambda_{\max }=319 \mathrm{~nm}$ and compared to a standard curve of the drug solution.
Cumulative drug release was calculated in terms of the ratio of the cumulative mass of the released drug at a given time against the initial drug loading in the blend microparticles. In vitro drug release tests were performed in triplicate $(n=3)$.

\section{RESULTS AND DISCUSSION}

\section{Morphology and size of blend microparticles}

The yield of the blend microparticles was in the range $85-92 \%$. The morphology of the blend microparticles was determined from their SEM micrographs as illustrated in Fig. 1. All blend microparticles were observed to have fine dispersibility, and the microparticles did not aggregate. The results suggest that the preparation conditions, including polymer concentration, volumes of oil and water phases, stirring speed and evaporation time for the oil-in-water emulsion solvent evaporation method used in this work were appropriate for preparing the drug-loaded blend microparticles with a high yield and good dispersibility.

Fig. 2 shows the surfaces of the blend

Table 1: Particle size and drug loading of the blend microparticles.

\begin{tabular}{|c|c|c|c|c|}
\hline PDLL/stearic acid (w/w) & $D_{\text {theoretical }}(\%)$ & $\mathrm{T}_{\mathrm{g}}^{\mathrm{a}}\left({ }^{\circ} \mathrm{C}\right)$ & $\mathrm{T}_{\mathrm{m}}{ }^{\mathrm{b}}\left({ }^{\circ} \mathrm{C}\right)$ & $\Delta H_{m}{ }^{b}(J / g)$ \\
\hline $100 / 0$ & 10 & 34 & - & - \\
\hline $97.5 / 2.5$ & 10 & 27 & 54 & 5.7 \\
\hline $95 / 5$ & 10 & 27 & 56 & 9.1 \\
\hline $92.5 / 7.5$ & 10 & 26 & 54 & 15.8 \\
\hline $97.5 / 2.5$ & 5 & 26 & 55 & 14.6 \\
\hline $97.5 / 2.5$ & 2.5 & 26 & 54 & 15.4 \\
\hline
\end{tabular}

\begin{tabular}{lcccc}
\hline $\begin{array}{l}\text { PDLL/stearic } \\
\text { acid }(\mathbf{w} / \mathbf{w})\end{array}$ & $\begin{array}{c}\text { Average particle } \\
\text { size }(\boldsymbol{\mu m}) \mathbf{( \% )}\end{array}$ & $\begin{array}{c}\text { DLC }_{\text {theoretical }} \\
\mathbf{( \% )}\end{array}$ & $\begin{array}{c}\mathbf{D L C}_{\text {actual }} \\
\mathbf{( \% )}\end{array}$ & DLE \\
\hline $100 / 0$ & $167 \pm 62$ & 10 & $6.7 \pm 1.5$ & 67 \\
$97.5 / 2.5$ & $175 \pm 79$ & 10 & $6.4 \pm 1.4$ & 64 \\
$95 / 5$ & $177 \pm 53$ & 10 & $6.6 \pm 1.5$ & 66 \\
$92.5 / 7.5$ & $167 \pm 58$ & 10 & $6.2 \pm 1.6$ & 62 \\
$97.5 / 2.5$ & $175 \pm 64$ & 5 & $3.2 \pm 1.1$ & 64 \\
$97.5 / 2.5$ & $168 \pm 75$ & 2.5 & $1.5 \pm 0.4$ & 60 \\
\hline
\end{tabular}

Table 2: DSC results of the blend microparticles 
microparticles. The resulting blend microparticles with PDLL/stearic acid blend ratios of $100 / 0$, 97.5/2.5 and 95/5 (w/w) were nearly spherical in shape with a smooth surface as shown in Figs. $1(\mathrm{a})-1(\mathrm{c})$ and 2(a)-2(c), respectively. This indicates that the PDLL/stearic acid blend ratio in this range did not affect the morphology of the microparticles. However, the 92.5/7.5 (w/w) PDLL/stearic acid blend microparticles were irregular in shape with a rough surface as shown in Figs. 1(d) and 2(d), respectively. This may be explained by the steraric acid molecules exhibiting self-aggregate to induce irregular microparticles and a rough surface for the 92.5/7.5 blend microparticles.

The average particle size of the blend microparticles was determined by light scattering analysis. All of the particle size graphs exhibited similar unimodal particle size distributions, an example of which is shown in Fig. 3 for the 92.5/7.5 (w/w) PDLL/stearic acid blend microparticles. The average particle size of the blend microparticles, as summarized in Table 1, was in the range of 167-177 $\mu \mathrm{m}$. The PDLL/stearic acid blend ratio did not affect the average particle size.

\section{Thermal properties of blend microparticles}

The thermal transition properties of the blend microparticles were investigated by DSC. The DSC thermograms of the blend microparticles are compared in Fig. 4. The DSC results are summarized in Table 2. The DSC thermogram of PDLL microparticles in Fig. 4(a) showed a single glass transition temperature $\left(\mathrm{T}_{\mathrm{g}}\right)$ at $34^{\circ} \mathrm{C}$ without a
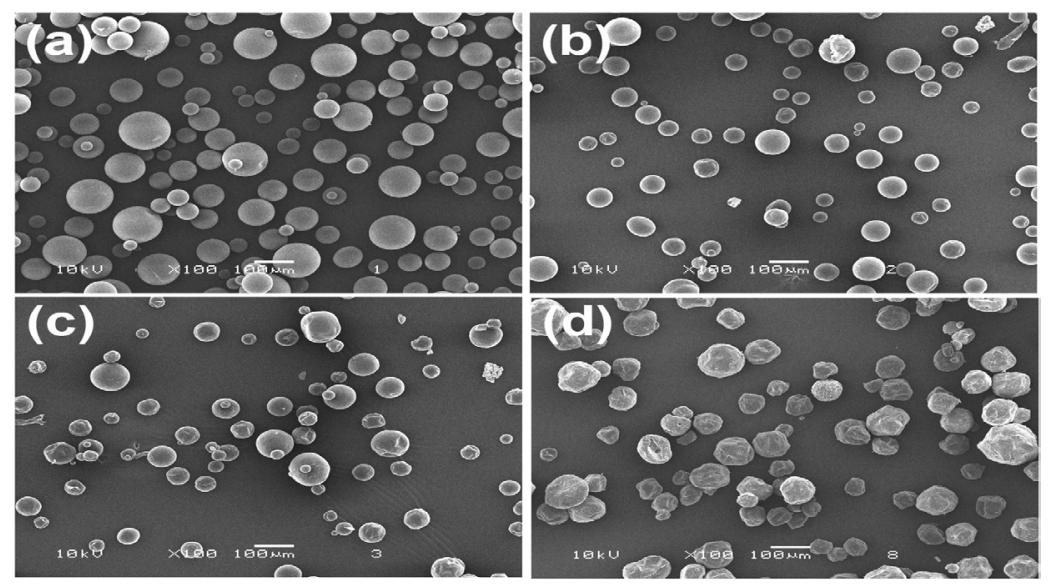

Fig. 1: SEM micrographs of the blend microparticles with PDLL/stearic acid blend ratios of (a) 100/0, (b) 97.5/2.5, (c) 95/5 and (d) 92.5/7.5 (w/w). All bars $=100 \mathrm{~mm}$

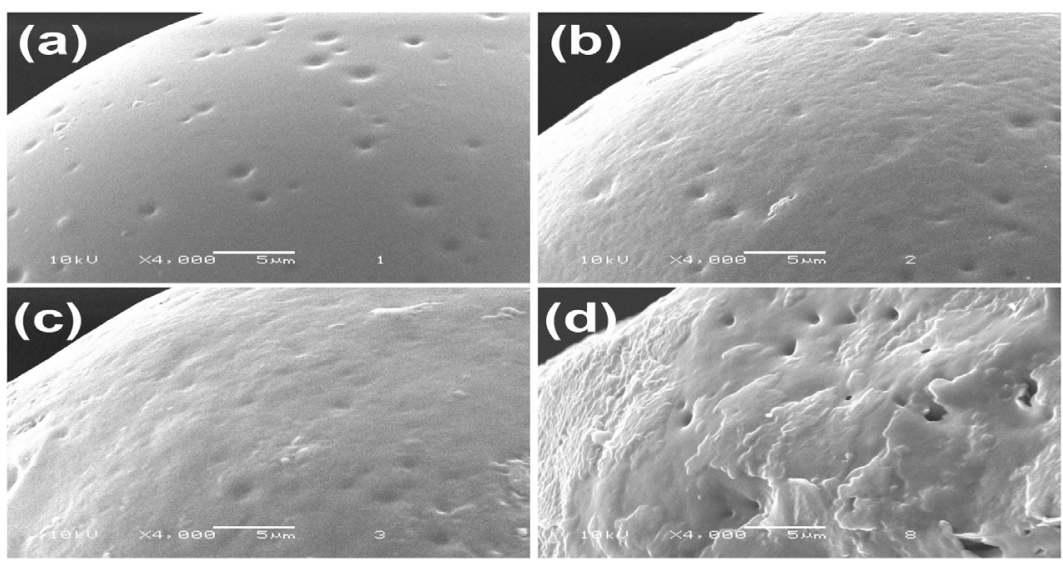

Fig. 2: Surfaces of the blend microparticles with PDLL/stearic acid blend ratios of (a) 100/0, (b) $97.5 / 2.5$, (c) $95 / 5$ and (d) $92.5 / 7.5$ (w/w). All bars $=5 \mu \mathrm{m}$ 


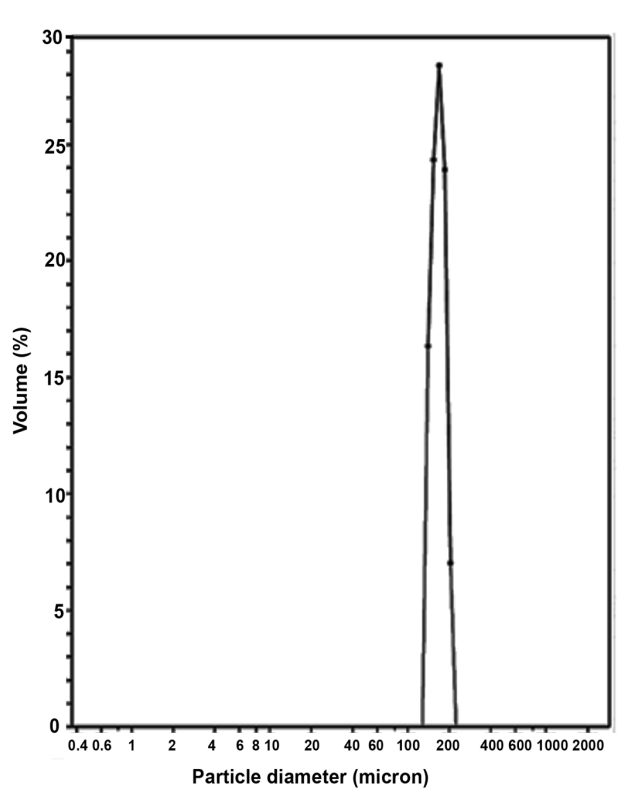

Fig. 3: Particle size graph for the $92.5 / 7.5$ (w/w) PDLL/stearic acid blend microparticles melting peak. The PDLL is amorphous. The blend microparticles exhibited a lower $\mathrm{T}_{g}\left(26-27^{\circ} \mathrm{C}\right)$ than the PDLL microparticles. This indicates that the PDLL and stearic acid were partial miscible. The blend microparticles also showed a single melting temperature $\left(T_{m}\right)$ in the range $54-56^{\circ} \mathrm{C}$ due to stearic acid crystallization. The stearic acid showed $\mathrm{a}_{\mathrm{m}}$ at $54^{\circ} \mathrm{C}$ (data did not shown). In addition, the heat of melting $\left(\mathrm{DH}_{\mathrm{m}}\right)$ for the blend microparticles, which was directly related to stearic acid crystallinity, decreased as the stearic acid ratio increased, which supports the partial miscibility between the PDLL and stearic acid phases.

\section{Drug loading and in vitro drug release of blend microparticles}

The theoretical drug loading content $\left(\mathrm{DLC}_{\text {theoretical }}\right)$, actual drug loading content (DLC $\left.\mathrm{actual}_{1}\right)$ and drug loading efficiency (DLE) calculated from equations (1) - (3), respectively are also summarized in Table 1. It was found that the PDLL microparticle

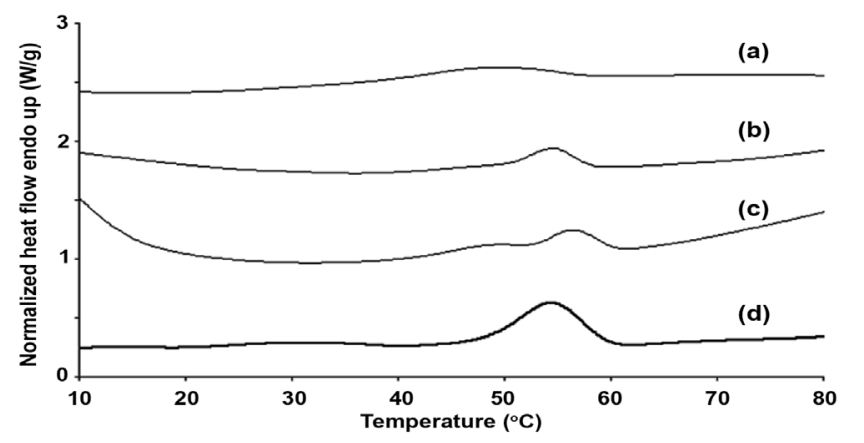

Fig. 4: DSC thermograms of the blend microparticles with PDLL/stearic acid blend ratios of (a) 100/0, (b) $97.5 / 2.5$, (c) $95 / 5$ and (d) $92.5 / 7.5$ (w/w)

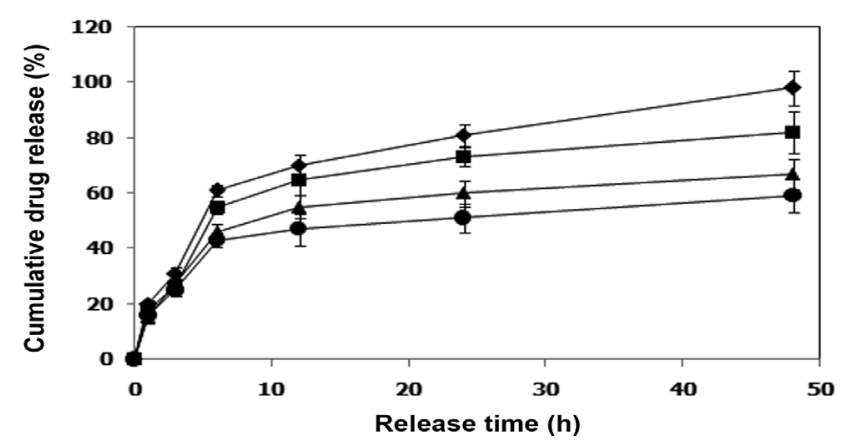

Fig. 5: Cumulative release of the indomethacin model drug from the blend microparticles prepared with PDLL/stearic acid blend ratios of $(\diamond) 100 / 0,(\square) 97.5 / 2.5$,

$(\Delta)$ 95/5 and () $92.5 / 7.5(w / w)$ for a drug loading content of $10 \%$ 


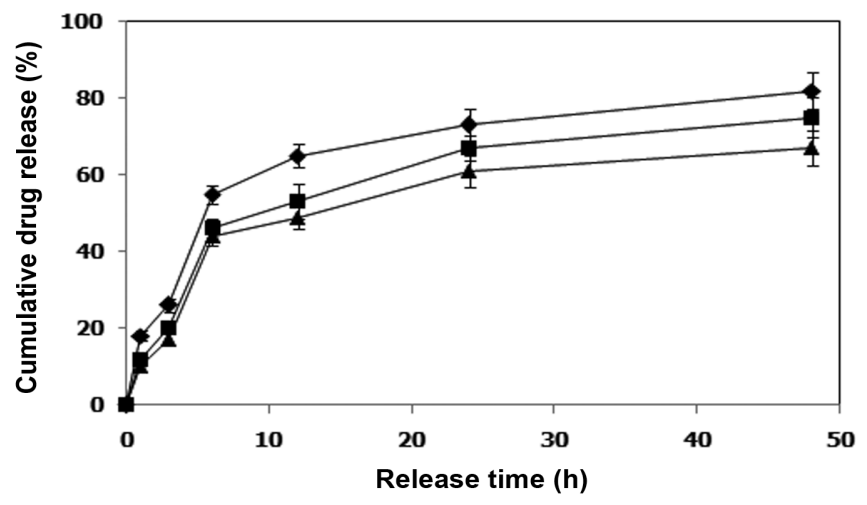

Fig. 6: Cumulative release of the indomethacin model drug from the blend microparticles prepared with drug loading contents of ( $) 10 \%$, (घ) $5 \%$ and $(\Delta) 2.5 \%$ for the PDLL/stearic acid blend ratio of $97.5 / 2.5(\mathrm{w} / \mathrm{w})$

sample showed the highest DLE (77\%). All blend microparticles showed similar DLE values in the range $60-66 \%$. This may be explained by the interactions of the stearic acid-indomethacin model drug that were weaker than the PDLL-indomethacin interactions during the microparticle formation. The DLC $_{\text {theoretical }}$ value did not significantly affect the DLE value.

The in vitro drug release of the indomethacin from the blend microparticles was determined in a phosphate buffer $\mathrm{pH} 7.4$ at $37^{\circ} \mathrm{C}$ for $48 \mathrm{~h}$. The influences of the PDLL/stearic acid blend ratio and $\mathrm{DLC}_{\text {theoretical }}$ value on the drug release behavior of the blend microparticles are illustrated in Figs. 5 and 6 , respectively. The drug release profile is plotted between cumulative drug release and release time. An initial burst release effect within the first $6 \mathrm{~h}$ was observed followed with a further sustained release for all microparticles. The initial burst release occurred due to the immediate release of the drug on and near the microparticle surfaces.

As shown in Fig. 5, the total cumulative drug release at $48 \mathrm{~h}$ decreased from $98 \%$ to $59 \%$ when the stearic acid ratio was increased from 0 to $7.5 \%$. This may be due to the hydrophobicity of the stearic acid component. The total cumulative drug release at $48 \mathrm{~h}$ also decreased from $82 \%$ to $67 \%$ when the DLC $_{\text {theoretical }}$ was decreased from $10 \%$ to $2.5 \%$ as shown in Fig. 6. This may be explained by the high drug loading content that could affect the properties of the polymeric network in the microparticle matrix, thus affecting the diffusion barrier. The results of the drug release demonstrated that the drug release from the PDLL/stearic acid blend microparticles was controlled by the PDLL/stearic acid blend ratio and drug loading content.

\section{CONCLUSION}

The present work showed that drug-loaded PDLL/stearic acid blend microparticles with various blend ratios could be successfully prepared by the oilin-water emulsion solvent evaporation method. The microparticles with PDLL/stearic acid blend ratios in the range of $100 / 0-95 / 5(\mathrm{w} / \mathrm{w})$ were spherical in shape and had smooth surfaces, but the 92.5/7.5 (w/w) blend microparticles were not. Particle size and drug loading efficiency of the PDLL and all the blend microparticles were similar. The thermal transition properties of the blend microparticles strongly depended upon the blend ratio. The release of indomethacin from the blend microparticles can be tailored by adjusting the PDLL/stearic acid blend ratio and drug loading content. These blend microparticles are considered to be promising drug carriers for controlled release of poorly water-soluble drugs.

\section{ACKNOWLEDGEMENTS}

This research was financially supported by Mahasarakham University, Mahasarakham, Thailand. Financial support from the Center of Excellence for Innovation in Chemistry (PERCH-CIC), Office of the Higher Education Commission, Ministry of Education, Thailand is also gratefully acknowledged. 


\section{REFERENCES}

1. Mohammad, S., Fatemeh, S., Mojgan, Y. Controlled release of acetaminophen from CMC-based hydrogels. Orient. J. Chem. 27: 895-902 (2011).

2. Kumar, V., Prajapati, S.K., Soni, G.C., Singh, M., Kumar, N. Sustained release matrix type drug delivery system: a review. World J. Pharm. Pharm. Sci. 1: 934-60 (2012).

3. Brannon-Peppas, L. Recent advances on the use of biodegradable microparticles and nanoparticles in controlled drug delivery. Int. J. Pharm. 116: 1-9 (1995).

4. Chung, T.W., Huang, Y.Y., Liu, Y.Z. Effects of the rate of solvent evaporation on the characteristics of drug loaded PLLA and PDLLA microspheres. Int. J. Pharm. 212: 161-169 (2001).

5. Cui, F., Cun, D., Tao, A., Yang, M., Shi, K., Zhao, M. Preparation and characterization of melittin-loaded poly(DL-lactic acid) or poly(DLlactic acid-co-glycolic acid) microspheres made by the double emulsion method. J. Controlled Release 107: 310-319 (2005).

6. Liu, M., Zhou, Z., Wang, X., Xu, J., Yang, K., Cui, Q. Formation of poly(L,D-lactide) spheres with controlled size by direct dialysis. Polymer 48: 5767-5779 (2007).

7. Baimark, Y., Srisa-ard, M. Preparation of drug-loaded microspheres of linear and star-shaped poly(D,L-lactide)s and their drug release behaviors. J. Appl. Polym. Sci. 124: 3871-3878 (2012).

8. Jain, R.A. The manufacturing techniques of various drug loaded biodegradable poly(lactide-co-glycolide) (PLGA) devices. Biomaterials 21:2475-2490 (2000).

9. Freiberg, S., Zhu, X.X. Polymer microspheres for controlled drug release. Int. J. Pharm. 282: 1-18 (2004).

10. Dong, Y., Feng, S.S. Nanoparticles of poly(D,L-lactide)/methoxy poly(ethylene glycol)-poly(D,L-lactide) blends for controlled release of paclitaxel. J. Biomed. Mater. Res. 78A: 12-19 (2006).

11. Muller, R.H., Mader, K., Gohla, S. Solid lipid nanoparticles (SLN) for controlled drug delivery - a review of the state of the art. Euro. J. Pharm. Biopharm. 50: 161-177 (2000).

12. M.Z.H. Rozaini, Orient J. Chem., 28(2): 803808 (2012).

13. Jaspart, S., Bertholet, P., Piel, G., Dogne, J.M., Delattre, L., Evrard, B. Solid lipid microparticles as a sustained release system for pulmonary drug delivery. Euro. J. Pharm. Biopharm. 65: 47-56 (2007).

14. Perge, L., Robitzer, M., Guillemot, C., Devoisselle, J.M., Quignard, F., Legrand, P. New solid lipid microparticles for controlled ibuprofen release: formulation and characterization study. Int. J. Pharm. 422 : 59-67 (2012).

15. Dalpiaz, A., Mezzena, M., Scatturin, A., Scalia, S. Solid lipid microparticles for the stability enhamcement of the polar drug cyclopentyladenosine. Int. J. Pharm. 355:8186 (2008). 